\title{
DEVELOPING ORGANIC AND INORGANIC NANOMEDICINE FOR CANCER THERAPY
}

\author{
Xinwei Cheng* \\ Department of Biomedical Engineering, The Ohio State University, Columbus, OH 43210, USA
}

\begin{abstract}
The application of nanomedicines has proved to be effective for the therapy of various types of cancer compared to conventional treatments such as chemotherapy and radiation therapy. The nanomedicines can treat various cancers with the precise target without affecting the normal cells. In addition, by utilizing of enhanced permeability and retention (EPR) effect, nanomedicines can selectively targeting disease site and yield very promising therapeutic results for cancer treatment. Among all available nanomedicines, organic materials such as polymers are widely used in recent decades as biomaterials for their favorable properties like excellent biocompatibility, easy design and preparation, several types of structures and interesting biomimetic character, which warrants the further evaluations on these kinds of innovative material. Inorganic nanoparticles such nanogold also demonstrated the ability to target cancer and can be employed as both therapeutic and imaging agents. This mini-review will give a brief discussion of using inorganic and organic nanoparticles for the treatment of cancer.
\end{abstract}

Keywords: Nanomedicine, self-assembly, drug delivery, nanoparticle, nanogold, anticancer drugs

Article Info

Received 11 Dec 2016; Review Completed 21 Dec 2016; Accepted 22 Jan 2017, Available online 15 March 2017

Cite this article as:

Cheng X, Developing organic and inorganic nanomedicine for cancer therapy, Journal of Drug Delivery and Therapeutics. 2017;

7(2):1-4. DOI: http://dx.doi.org/10.22270/jddt.v6i6.1367

*Address for Correspondence

Department of Biomedical Engineering, The Ohio State University, Columbus, OH 43210, USA; Tel.: 614-286-3918.

E-mail: cheng.726@buckeyemail.osu.edu

\section{INTRODUCTION}

Cancer has become one of the most deadly diseases due to its fast abnormal cell growth. Change in the DNA sequence by mutation and thereby altering the genetic expression is the root cause of cancer. This mutation could result from several external factors like exposure to UV light or other carcinogenic compounds like tobacco. DNA methylation occurs because of the mutation, in which a methyl group is added to the cytosine and adenine of DNA.

At present, chemotherapy is the most common and widely used treatment for cancer ${ }^{1-4}$. Nanomedicines, use the latest nanotechnology for many medical applications ${ }^{5-7}$. According to Heim \& Mitelman ${ }^{8}$, nanomedicines have been proven effective in treating and preventing cancer compared to other forms of treatments ${ }^{9}$. The drugs used in the nanomedicines are prepared in nanometers and thus can be directed to the site of cancer accurately without many side effects ${ }^{10,11}$.

\section{CURRENTLY USED METHODS FOR TREATING CANCER}

In order to treat cancer effectively, it is quite necessary to diagnose cancer at the early stage ${ }^{12}$. Due to the relatively immature diagnose technique, it is hard to detect the cancer at an early stage ${ }^{13}$. Fortunately, there are multiple potent therapeutic techniques available for cancer treatment.

Surgery is one of the most common approaches used for removing the tumor at an early stage, which prevents the tumor cells from spreading to other normal organs. Chemotherapy, radiation therapy, hormonal therapy, and immunotherapy are conventional therapies for cancer treatment ${ }^{14}$. During chemotherapy, many anti-cancer drugs, such as docetaxel, doxorubicin, cyclophosphamide, etc, are administered into the human body to inhibit cell proliferation and metastasis ${ }^{15}$. Depending on the different characteristics of cancer, various drugs are applied to the patients during the treatment. This kind of therapy is usually 
combined with some other form of treatments, such as radiation therapy to improve the efficacy.

The effectiveness of this treatment also depends on the type of cancer and what stage of cancer has entered ${ }^{16}$. Flygare et al believed that if the treatment has been given at an early stage, .cancer would be sensitive to many kinds of treatments ${ }^{17}$. Leukemia, a type of cancer occurring in the bone marrow, which causes a rise in the number of white blood cells, can be treated with chemotherapy with a good outcome. However, during the treatment of brain and skin tumors, chemotherapy has not been reported to be very effective ${ }^{18}$. This may due to the poor permeability of Blood- Brain Barrier (BBB) and the of drug resistance produced by p-glycoprotein.

In addition, it is essential to have the "perfect" dosage of the drugs that are incorporated into chemotherapy ${ }^{19}$. As these drugs are very toxic and efficient, overdosage can be very harmful to normal organs of cancer suffering patient. While low dosage may not even have effects on the cancerous cells and tissues. Therefore, it is significant to have this "perfect" drug dosage, which highly depends on the stage of cancer, body surface area, body mass, height, and age of a patient ${ }^{20}$. It has been reported that the chemotherapy often related to some side effects without the targeting, which will cause damage to other normal organs $^{21}$. In most circumstances, patient receiving this treatment often suffer from a headache, nausea diarrhea, constipation and abdominal pain. Moreover, the immune system is usually impaired by this nontargeting effect shown as the vastly decreasing white blood cells. Hair loss is another common side effect resulted from the inhibition of hair cells from dividing normally.

Radiation therapy is another technique that can be applied to the treatment of cancer. Briefly, the cancerous cells are exposed to ionizing radiation that can potentially kill those cells. According to $\mathrm{Mi}^{22}$, this therapy is effective if the cancer is localized to a restricted area without metastasis. This therapy causes damage to the DNA of the cancerous cells and thereby inhibits the cell proliferation.

Radiation therapy can be painless, which is different from chemotherapy. Nevertheless, common side effects, such as nausea, damage to the epithelial tissues, and infertility, are still presented.

\section{USEFULNESS OF NANOMEDICINES FOR CANCER THERAPY BASED ON EPR EFFECT}

The Enhanced Permeability and Retention (EPR) effect is a unique property. To be more specific, due to the differences of pathophysiological and anatomical in solid tumors, nanosize drugs tend to leak out of the loose tumor blood vessel easily and accumulate in solid tumors ${ }^{23}$. The EPR based cancer treatment has become highly effective and offered a broad range of potentiality to the patients ${ }^{24}$.
The side effects are similar to other common cancer treatment ${ }^{25}$. According to $\mathrm{Cha}^{26}$, the small sizes of nanomedicines with targeting ligand facilitate their binding to the cancerous cells accurately and thus can yield satisfactory result compared to conventional treatments.

The tumor cells have their own characteristics. They are able to attract the blood vessels to provide nutrition and oxygen, which will boost angiogenesis. In addition, tumors have a poor lymphatic drainage system that leads to the variable retention rate produced by high interstitial pressure. Due to these merits, nanomedicines often easily leak out of the blood vessel and enter the actual tumor tissues ${ }^{27}$. Eventually, because of the high retention rate of the tumor, once the tumor tissues have taken up the nanomedicines, it is tough to escape from the tumor again ${ }^{28}$.

\section{USE OF POLYMER NANOPARTICLE FOR TREATING CANCER}

In the field of smart drug delivery, polymer plays an important role, in its ability to deliver therapeutic agents directly to the site of action with high efficiency ${ }^{29,30}$. Thus, the delivery system of the smart drug has become successful by the application of advanced polymer science in the field of nanobiotechnology $y^{31,32}$. It possesses some valuable features, such as sustained and controlled release, targeted delivery ${ }^{33,34}$. There are several micro-scale and nano-scale sized particles that are utilized in biological applications ${ }^{35-38}$.

In addition, many polymeric nanoparticle delivery systems are used in the therapeutic treatment of cancer ${ }^{39-41}$. According to Kim et $\mathrm{al}^{42}$, the properties of the nanoparticle systems need to be adjusted according to the characteristics of the tumor. The types of nanoparticle systems explored for applications in cancer therapy include the dendrimers, polymers as well as the polymeric nanoparticles. The increasing number of interests in modifying the polymeric chemical systems gives them a direction for the targeted and therapeutic aspects ${ }^{43}$. Several polymeric systems have been developed into therapeutic platforms, which include the solid polymeric nanoparticles and polymeric micelle. The function of the nanoparticles was to assist achieving the extended circulation time of drug and reducing the nonspecific tissue distribution. The negatively charged nanoparticles are more efficient than the positively charged ones and the size of these particles range from 1-100 $\mathrm{nm}$ that help to increase the phagocytic capability of the cell and thereby enhancing the drug delivery system.

\section{USE OF GOLD NANOPARTICLE FOR CANCER THERAPY}

Nanoparticles have been developed as it possesses valuable impact on the treatment of various diseases $^{44-46}$. It has been reported that gold nanoparticles are able to absorb a certain wavelength of light and produce greatly increased temperature ${ }^{47}$. This is one of the very effective thermal therapies. Due to their increased atomic mass, the absorption wavelength 
can also shift. This is quite different from the soft tissue cells that are not able to absorb the extensive amount of radiation during the treatment. Thus, as per Hainfeld et al stated, an increase in the dose of radiation is possible using the gold nanoparticles for treating tumors and cancers when gold nanoparticles are heated $u^{48}$.

In the field of nanotechnology, nanoparticles are optimized on their surface, size, zeta and the inner phase to treat various types of cancer ${ }^{49}$. With specific surface medication, drugs have increased their potential in targeting the site of action without causing much side effects. As stated by Maeda et $\mathrm{al}^{33}$, a 5-nm gold nanoparticle has been conjugated to cetuximab covalently along with gemcitabine for the treatment of pancreatic cancer. In order to decrease the possibility of affect normal healthy cells during the treatment, gold nanoparticles are usually coated with an antibody that is able to recognize specific receptor on target cancer cell. According to Alhasan et $\mathrm{al}^{50}$, the particle size of PEGcoated gold nanoparticles around $130 \mathrm{~nm}$ in diameter are observed to be utilized for treating cancer-based on the EPR effect ${ }^{51}$. Effect of gold nanoparticles during cancer treatment is evaluated based on the color and distance. It is generally believed that gold nanoparticles are not toxic, which enhances their use in the various treatment processes ${ }^{52}$. Also, gold nanoparticles have also been employed as imaging modalities to detect cancer cells due to the enhanced scattering ability upon absorbing of radiation. Another positive impact of gold nanoparticles is by utilizing antibody for Epidermal Growth Factor Receptor (EFGR), which is a protein

\section{REFERENCES}

(1) Dave, R. H.; Shah, D. A.; Patel, P. G. Development and evaluation of high loading oral dissolving film of aspirin and acetaminophen. Journal of Pharmaceutical Sciences and Pharmacology 2014, 1, 112-122.

(2) Guo, X. K.; Sun, H. P.; Shen, S.; Sun, Y.; Xie, F. L.; Tao, L.; Guo, Q. L.; Jiang, C.; You, Q. D. Synthesis and evaluation of gambogic acid derivatives as antitumor agents. Part III. Chemistry \& biodiversity 2013, 10, 73-85.

(3) Liu, Z.-1.; Zhang, R.-m.; Meng, Q.-g.; Zhang, X.-c.; Sun, Y. Discovery of new protein kinase CK2 inhibitors with 1,3dioxo-2,3-dihydro-1H-indene core. MedChemComm 2016, 7, 1352-1355.

(4) Sun, H.; Zhu, J.; Chen, Y.; Sun, Y.; Zhi, H.; Li, H.; You, Y.; Xiao, Q. Docking Study and Three- Dimensional Quantitative Structure- Activity Relationship (3D- QSAR) Analyses and Novel Molecular Design of a Series of 4- Aminoquinazolines as Inhibitors of Aurora B Kinase. Chinese Journal of Chemistry 2011, 29, 1785-1799.

(5) Cheng, X.; Lee, R. J. The role of helper lipids in lipid nanoparticles (LNPs) designed for oligonucleotide delivery. Advanced drug delivery reviews 2016, 99, 129-137.

(6) Cheng, X.; Liu, Q.; Li, H.; Kang, C.; Liu, Y.; Guo, T.; Shang, K.; Yan, C.; Cheng, G.; Lee, R. J. Lipid Nanoparticles Loaded with an Antisense Oligonucleotide Gapmer Against Bcl-2 for Treatment of Lung Cancer. Pharmaceutical Research 2016, 1-11.

(7) Wicki, A.; Witzigmann, D.; Balasubramanian, V.; Huwyler, J. Nanomedicine in cancer therapy: challenges, opportunities, and clinical applications. Journal of Controlled Release 2015, 200, 138-157.

(8) Heim, S.; Mitelman, F.: Cancer cytogenetics: chromosomal and molecular genetic aberrations of tumor cells; John Wiley \& Sons, 2015. that expressed on the surface of cancer cells. This facilitates the interaction with cancer cells other than the normal cells. It has been reported that gold nanoparticles have the ability to heat the cancer cells in a rapid manner compared to the normal cells based on the property of heat-conductor exhibited by gold $^{42}$. Eventually, Gold nanoparticles with cell penetrating peptide enhance their ability to be uptake by the cancer cells, which will essentially increase their accumulation in the tumor based on the EPR effect.

\section{CONCLUSION}

The application of nanomedicines has proved to be effective for cancer treatment compared to conventional chemotherapy and radiation therapy. The nanomedicines can treat various cancers with the precise target without affecting the normal cells. In addition, by utilizing of enhanced permeability and retention (EPR) effect, nanomedicines can yield a very promising result for cancer treatment.

Moreover, polymers are widely used in recent decades as biomaterials for their favorable properties like excellent biocompatibility, easy design and preparation, several types of structures and interesting biomimetic character, which warrants the further evaluations on these kinds of innovative materials.

\section{ACKNOWLEDGEMENTS}

The author thanks Dr. Robert J. Lee and Dr. Chen Kang for the valuable scientific discussion.

(9) Yung, B. C.; Li, J.; Zhang, M.; Cheng, X.; Li, H.; Yung, E. M.; Kang, C.; Cosby, L. E.; Liu, Y.; Teng, L. Lipid Nanoparticles Composed of Quaternary Amine-Tertiary Amine Cationic Lipid Combination (QTsome) for Therapeutic Delivery of AntimiR-21 for Lung Cancer. Molecular pharmaceutics 2016, 13, 653-662.

(10) Stacey, J.: Teratologies: A cultural study of cancer; Routledge, 2013.

(11) Ding, R.; Yu, X.; Wang, P.; Zhang, J.; Zhou, Y.; Cao, X.; Tang, H.; Ayres, N.; Zhang, P. Hybrid photosensitizer based on amphiphilic block copolymer stabilized silver nanoparticles for highly efficient photodynamic inactivation of bacteria. RSC Advances 2016, 6, 20392-20398.

(12) Chen, Y.; Bian, Y.; Sun, Y.; Kang, C.; Yu, S.; Fu, T.; Li, W.; Pei, Y.; Sun, H. Identification of 4-aminoquinoline core for the design of new cholinesterase inhibitors. PeerJ 2016, 4, e2140

(13) Butterworth, K. T.; Coulter, J.; Jain, S.; Forker, J.; McMahon, S.; Schettino, G.; Prise, K.; Currell, F.; Hirst, D. Evaluation of cytotoxicity and radiation enhancement using $1.9 \mathrm{~nm}$ gold particles: potential application for cancer therapy. Nanotechnology 2010, 21, 295101.

(14) Waller, A. P.; George, M.; Kalyanasundaram, A.; Kang, C.; Periasamy, M.; Hu, K.; Lacombe, V. A. GLUT12 functions as a basal and insulin-independent glucose transporter in the heart. Biochimica et Biophysica Acta (BBA)-Molecular Basis of Disease 2013, 1832, 121-127.

(15) Sun, Y.; Wang, H.-p.; Zhou, Y.; YOU, Q.-d. Recent advances in non-campto-thecin DNA topoisomerase I inhibitors as anticancer drugs. Prog Pharm Sci 2011, 35, 385-395.

(16) Zhou, Y.; Xu, X.; Sun, Y.; Wang, H.; Sun, H.; You, Q. Synthesis, cytotoxicity and topoisomerase II inhibitory activity of lomefloxacin derivatives. Bioorganic \& medicinal chemistry letters 2013, 23, 2974-2978. 
(17) Flygare, J. A.; Pillow, T. H.; Aristoff, P. Antibody- drug conjugates for the treatment of cancer. Chemical biology \& drug design 2013, 81, 113-121.

(18) Wildiers, H.; Mauer, M.; Pallis, A.; Hurria, A.; Mohile, S. G.; Luciani, A.; Curigliano, G.; Extermann, M.; Lichtman, S. M.; Ballman, K. End points and trial design in geriatric oncology research: a joint European organisation for research and treatment of cancer-Alliance for Clinical Trials in OncologyInternational Society Of Geriatric Oncology position article. Journal of Clinical Oncology 2013, 31, 3711-3718.

(19) Murdande, S. B.; Shah, D. A.; Dave, R. H. Impact of nanosizing on solubility and dissolution rate of poorly soluble pharmaceuticals. Journal of pharmaceutical sciences 2015, 104, 2094-2102.

(20) Han, R.; Sun, Y.; Kang, C.; Sun, H.; Wei, W. Amphiphilic dendritic nanomicelle-mediated co-delivery of 5-fluorouracil and doxorubicin for enhanced therapeutic efficacy. Journal of Drug Targeting 2016.

(21) Song, L.; Kang, C.; Sun, Y.; Huang, W.; Liu, W.; Qian, Z. Crocetin Inhibits Lipopolysaccharide-Induced Inflammatory Response in Human Umbilical Vein Endothelial Cells. Cellular Physiology and Biochemistry 2016, 40, 443-452.

(22) Mi, Y.; Zhao, J.; Feng, S.-S. Targeted co-delivery of docetaxel, cisplatin and herceptin by vitamin E TPGScisplatin prodrug nanoparticles for multimodality treatment of cancer. Journal of controlled release 2013, 169, 185-192.

(23) Shah, D. A.; Murdande, S. B.; Dave, R. H. A Review: Pharmaceutical and Pharmacokinetic Aspect of Nanocrystalline Suspensions. Journal of Pharmaceutical Sciences 2016, 105, 10-24.

(24) Sun, Y.; Kang, C.; Zhang, A.; Liu, F.; Hu, J.; Zhong, X.; Xie, J. Co-delivery of dual-drugs with nanoparticle to overcome multidrug resistance. European Journal of BioMedical Research 2016, 2, 12-18.

(25) Du, J.; Cullen, J. J.; Buettner, G. R. Ascorbic acid: chemistry, biology and the treatment of cancer. Biochimica et Biophysica Acta (BBA)-Reviews on Cancer 2012, 1826, 443457.

(26) Cha, E.; Klinger, M.; Hou, Y.; Cummings, C.; Ribas, A.; Faham, M.; Fong, L. Improved survival with T cell clonotype stability after anti-CTLA-4 treatment in cancer patients. Science translational medicine 2014, 6, 238ra270-238ra270.

(27) Shah, D. A.; Patel, M.; Murdande, S. B.; Dave, R. H. Influence of spray drying and dispersing agent on surface and dissolution properties of griseofulvin micro and nanocrystals. Drug development and industrial pharmacy 2016, 1-9.

(28) Dawidczyk, C. M.; Russell, L. M.; Searson, P. C. Nanomedicines for cancer therapy: state-of-the-art and limitations to pre-clinical studies that hinder future developments. Frontiers in chemistry 2014, 2, 69.

(29) Kang, C.; Sun, Y.; Zhu, J.; Li, W.; Zhang, A.; Kuang, T.; Xie, J.; Yang, Z. Delivery of Nanoparticles for Treatment of Brain Tumor. Current Drug Metabolism 2016, 17, 745-754.

(30) Yu, X.; Chen, X.; Chai, Q.; Ayres, N. Synthesis of polymer organogelators using hydrogen bonding as physical crosslinks. Colloid and Polymer Science 2016, 294, 59-68.

(31) Korang-Yeboah, M.; Rahman, Z.; Shah, D.; Mohammad, A.; Wu, S.; Siddiqui, A.; Khan, M. A. Impact of formulation and process variables on solid-state stability of theophylline in controlled release formulations. International journal of pharmaceutics 2016, 499, 20-28.

(32) Korang-Yeboah, M.; Rahman, Z.; Shah, D. A.; Khan, M. A. Spectroscopic-Based Chemometric Models for Quantifying Low Levels of Solid-State Transitions in Extended Release Theophylline Formulations. Journal of pharmaceutical sciences 2016, 105, 97-105.

(33) Maeda, H.; Nakamura, H.; Fang, J. The EPR effect for macromolecular drug delivery to solid tumors: Improvement of tumor uptake, lowering of systemic toxicity, and distinct tumor imaging in vivo. Advanced drug delivery reviews 2013, 65, 71-79.
(34) Wang, L.; Maruf, S. H.; Maniglio, D.; Ding, Y. Fabrication and characterizations of crosslinked porous polymer films with varying chemical compositions. Polymer 2012, 53, 3749-3755.

(35) Li, H.; Cheng, X.; Liu, Y.; Lee, Y. B.; Kim, D. J.; Ahn, C.-h.; Lee, R. J. Folate receptor-targeted lipid coated albumin nanoparticles (F-LCAN) for therapeutic delivery of RX-0201 (Archexin ${ }^{\circledR}$ ), an antisense oligonucleotide against Akt-1. Cancer Research 2016, 76, 1322-1322.

(36) Sun, Y.; Kang, C. Self-Assembly of Peptides into Hydrogel. Journal of Organic \& Inorganic Chemistry 2016, 2.

(37) Wang, L.; Zhang, Z.; Ding, Y. Photocrosslinking-induced phase separation in evaporative solvents: formation of skin layers and microspheres. Soft Matter 2013, 9, 4455-4463.

(38) Yao, Z.; Sun, Y.; Kang, C. Structure and Self-Assembly of Multicolored Naphthalene Diimides Semiconductor. Nano LIFE 2016, 6, 1642007.

(39) Li, H.; Quan, J.; Zhang, M.; Yung, B. C.; Cheng, X.; Liu, Y.; Lee, Y. B.; Kim, D. J.; Lee, R. J. Lipid-albumin nanoparticles (LAN) for therapeutic delivery of antisense oligonucleotide against HIF-1alpha. Molecular pharmaceutics 2016.

(40) Sun, Y.; Kang, C.; Yao, Z.; Liu, F.; Zhou, Y. Peptide-Based Ligand for Active Delivery of Liposomal Doxorubicin. Nano LIFE 2016, 6, 1642004.

(41) Yang, Z.; Xie, J.; Zhu, J.; Kang, C.; Chiang, C.; Wang, X.; Wang, X.; Kuang, T.; Chen, F.; Chen, Z. Functional exosome-mimic for delivery of siRNA to cancer: in vitro and in vivo evaluation. Journal of Controlled Release 2016, 243, 160-171.

(42) Kim, M. S.; Lee, J.; Sidransky, D. DNA methylation markers in colorectal cancer. Cancer and Metastasis Reviews 2010, 29, 181-206.

(43) Hainfeld, J. F.; Dilmanian, F. A.; Zhong, Z.; Slatkin, D. N.; Kalef-Ezra, J. A.; Smilowitz, H. M. Gold nanoparticles enhance the radiation therapy of a murine squamous cell carcinoma. Physics in medicine and biology 2010, 55, 3045.

(44) Jin, Z.; Liu, X.; Duan, S.; Yu, X.; Huang, Y.; Hayat, T.; Li, J. The adsorption of Eu (III) on carbonaceous nanofibers: batch experiments and modeling study. Journal of Molecular Liquids 2016, 222, 456-462.

(45) Kang, C.; Sun, Y.; Wang, M.; Cheng, X. Nanosized Camptothecin Conjugates for Single and Combined Drug Delivery. European Journal of BioMedical Research 2016, 2 , 8-14.

(46) Sun, Y.; Kang, C.; Liu, F.; Song, L. Delivery of Antipsychotics with Nanoparticles. Drug Development Research 2016, 77, 393-399.

(47) Chai, Q.; Yu, X.; Jiao, Y. Applications of Gold Nanoparticles in Biosensors. Nano LIFE 2016.

(48) Hainfeld, J. F.; Smilowitz, H. M.; O'Connor, M. J.; Dilmanian, F. A.; Slatkin, D. N. Gold nanoparticle imaging and radiotherapy of brain tumors in mice. Nanomedicine 2013, 8, 1601-1609.

(49) Wang, T.; Jiao, Y.; Chai, Q.; Yu, X. Gold Nanoparticles: Synthesis and Biological Applications. Nano LIFE 2015, 5, 1542007.

(50) Alhasan, A. H.; Kim, D. Y.; Daniel, W. L.; Watson, E.; Meeks, J. J.; Thaxton, C. S.; Mirkin, C. A. Scanometric microRNA array profiling of prostate cancer markers using spherical nucleic acid-gold nanoparticle conjugates. Analytical chemistry 2012, 84, 4153-4160.

(51) Liu, F.; Sun, Y.; Kang, C.; Zhu, H. Pegylated Drug Delivery Systems: From Design to Biomedical Applications. Nano LIFE 2016, 6, 1642002.

(52) Hogberg, T.; Signorelli, M.; De Oliveira, C. F.; Fossati, R.; Lissoni, A. A.; Sorbe, B.; Andersson, H.; Grenman, S.; Lundgren, C.; Rosenberg, P. Sequential adjuvant chemotherapy and radiotherapy in endometrial cancer-results from two randomised studies. European Journal of Cancer 2010, 46, 2422-2431. 